\title{
An Experiment in Product Innovation and Design in a Mechanical Engineering Capstone Experience
}

\author{
Stephen M. Batill \\ University of Notre Dame
}

\begin{abstract}
Product design is inherently a collaborative, multidisciplinary activity that is influenced by numerous issues and it can be accomplished using many, very different approaches. This paper describes a number of pedagogical changes associated with a capstone design class in Mechanical Engineering that is intended to simulate the product development process as it occurs in industry. The changes from earlier offerings of this project-based, team-oriented course involved the type of corporate culture in which the design process took place, the nature of the product and an attempt to develop collaboration between students from engineering, marketing and design. The paper outlines the learning objectives for this course, its implementation and presents a preliminary assessment of the impact of the changes.
\end{abstract}

\section{Introduction and Overview of the Experiment}

The capstone design class in the Mechanical Engineering program at Notre Dame has undergone a number of changes in the past few years that have altered the types of projects and the associated technologies invoked by the students. The course is presented as a team-based, product-focused, design-build activity. It is a single semester, three-credit hour, course that is required of all students in the major and it is normally taken during the fourth year, as effectively all students complete their degree in four years. The students are organized into small designbuild teams and each team is responsible for the concept definition, design, documentation, prototype fabrication and evaluation of a mechanical system.

The projects are presented in a way that attempts to simulate the engineering design process. The key concept to be emphasized is that of simulating the design process as practiced in industry. Due to limits on time, the student's experience, financial resources and support facilities, it is often very difficult to engage students in projects that encompass all the important aspects of the product development process in the same detail that they will experience in industry. Though some programs have been able to successfully develop capstone design experiences that involve a direct industry interaction, this can be problematic particularly for single semester projects. ${ }^{1,2}$ There is a trade-off that exists between providing a realistic design environment, often associated with working with industry partners, and achieving the learning objectives set forth for a particular academic program.

Other programs have developed unique learning experiences that are designed to provide realistic capstone projects ${ }^{3-4}$ that also use the concept of simulation. In those cases where programs attempt to integrate other disciplines or concerns in the design process simulations, the manner in which those disciplines are included depends upon many factors including the faculty experiences, time, facilities and financial resources. It is well recognized that the engineering 
design process is a complex, multidisciplinary, collaborative activity and providing students with that perspective is an important learning objective.

For the past 5 years at Notre Dame the projects have involved the development of "products" that could be characterized as automated systems. These projects have required the students to engage a wide range of technical issues that include the use of sensors, actuators and embedded control. The products have ranged from autonomous seek-and-retrieve vehicles to automated packaging systems. In each case the basic product performance requirements were defined for the design teams and their efforts were focused on achieving the desired technical performance. The projects were selected to be consistent with the facilities provided within the design studio and available fabrication equipment. Due to the desire to integrate automation and control issues and recognizing the limited experience the mechanical engineering students have with embedded control technologies, the products were rather simple, though each presented significant technical challenges.

A common student response to the course was that it was a good experience but they were sometimes frustrated by the "simple" nature of the projects and they wanted to address products that were more "exciting." The aerospace engineering students in this combined AME department for many years have designed and built remotely or autonomously piloted aircraft and, at least from some student's viewpoint, these projects were more glamorous and engaging. Even though the fundamental learning objectives, technologies and student activities were identical in both capstone courses (the author has been involved with both of these programs) the students perceived a difference and it was almost exclusively based upon their perceived value of the product.

This paper describes a number of features introduced during the Fall 2001 term in order to address some of these concerns. Unlike the aircraft design program where the product design is often driven by complex, interacting technologies but the fundamental products (airplanes) are essentially the same, the products designed by mechanical engineers span the complete spectrum of human-made artifacts. Taking advantage of this feature and also introducing issues related to innovation and creativity were the key aspects of the "experiments" discussed herein.

To address these concerns, the fall 2001 offering of this course added a number of new features. Due to some scheduling issues, the enrollment during this semester was rather small (16 students in comparison with a normal enrollment of 30-40) and thus allowed for some experimentation and the introduction of new ideas. The first innovation involved the class of products being designed and the second the "corporate culture" in which the simulated, product-development process takes place. The second change was a direct result from the first. Once a decision was made to change the nature of the product, then the question arose as to what would be the best manner in which to conduct this simulation. Since the new products would require significant innovation and the students would be involved with defining the basic product as well as engineering its design, it appeared as if a process that enhanced and supported the innovation process was desirable.

In the Fall 2001 offering of this course, the students were tasked with designing "consumer products" for which they were required to define the product concept, its desirable attributes and 
demonstrate its technical feasibility. They were given the basic requirement to design an "interactive toy for the 2002 holiday season with a suggested retail cost of less than $\$ 100$." Obviously, designing "toys" has been used extensively in similar courses for many of the same reasons. The students can relate to toys as consumers and they generate a level of excitement sometimes not found in designing a new "clutch mechanism".

It was recognized that these changes would introduce concepts and issues that sometimes do not occur in the engineering curriculum such as marketing and product aesthetics, thus a collaboration was established to extend the scope of the project. To assist in those phases of this project related to concept evaluation, market definition and product styling, the engineering students teamed with students from the Mendoza College of Business Marketing Department and the College of Art and Letters Industrial Design programs. Students from the marketing class in Product Innovation assisted in the concept development by developing and performing market assessments. Students from the Digital Design class in the Industrial Design Program in the College of Arts and Letters developed 3-D visual renderings for the concepts to assist in market assessment and presentations. These interactions required the engineers to balance the technical, economic and aesthetic attributes of the product as well as to develop their ability to communicate with non-technical discipline specialists.

The second change involved the "corporate culture" or framework in which the project would be conducted. During the development of the ideas presented in this paper, the author had a number of communications with Ms. Tracy Lukawski, from IDEO, an internationally recognized design and product development firm. These discussions were prompted by the desire to use a consumer product as the focus for this course. The discussions and a chance encounter with Reference 5 prompted the second experiment related to corporate culture. The design teams were given the option of choosing between a framework that was structured similar to many product design processes in a variety of industries and organized by "management", or a framework based upon a less-structured, design studio environment based on the IDEO model.

In the structured process framework the project supervisor assigns the team members. There is a heavy emphasis on coordinated engineering analysis, modeling and simulation. Formalized engineering trade studies were used to validate elements of the design and the prototype was used to validate the engineering studies. The second framework involved the use of small, selfdirected, product-development studio teams. Since different teams selected different approaches, direct comparison of their experiences is included in the paper.

The following sections detail the results of these two changes and their influence on the overall learning objectives for this course. The impact is evaluated from the student's and instructor's perspectives and was assessed by evaluation of the documented results of the student's activities, self-assessment surveys of the students, and input from the industry review panel and faculty participants.

\section{Course Objectives}

The course documented in this paper is the capstone design experience in the Mechanical Engineering degree program. It is a single semester, 3-credit hour course that is required of all 
students in the major. It is usually taken in either the first or second semester of the $4^{\text {th }}$ year. The instructor's goals set forth for the course and presented to the students in the first class are:

- Provide an experience that is based upon active student participation.

- Enhance skills related to engineering design methodology, including modeling, simulation and parametric trade studies.

- Simulate the mechanical engineering product development process.

- Illustrate the interaction between competing technical and non-technical issues and the role of compromise, constraints and merit.

- Provide exposure to various phases of the design process, from definition of requirements to product realization.

- Help develop an understanding of the planning, coordination and communication required in a team effort.

An additional "goal" was added this year and that was to:

- Provide the opportunity for the students to innovate and express individual creativity.

The product design simulation described in this paper is structured in such a manner as to attempt to best achieve these goals. The course contains very little formal instruction and most of the student-faculty interaction is one-on-one. There are 6 hours each week of dedicated "design studio time" during which the students work with the instructor, graduate teaching assistants and technical consultants. The students are encouraged to try to exploit the technical skills developed in their previous courses as they develop concepts and work to demonstrate the technical feasibility of their ideas. Significant emphasis is placed upon:

- Gathering quantitative information to assist in the decision making process that is part of design.

- Written documentation of their engineering activities.

- Oral presentations of status and progress as well as developing the ability to ask effective questions to help evaluate the work of others,

- Developing a first-hand appreciation of the challenges associated with transitioning ideas to functioning devices.

As outlined below the students spend considerable time working with others to solve problems that they pose for themselves. Written documents are evaluated and returned, often for re-writes. All technical questions are answered to the best of the ability of the instructors. In almost all cases the solutions to the questions posed by the students are not "in the back of the book" so much time is spent developing engineering solutions to unique problems encountered during the concept development process. Emphasis is placed on developing the student's ability to pose, formalize, resolve and communicate the problems they encounter.

\section{Recognizing Differences in Corporate Culture and Product Development Processes}

A central element in the design process is making decisions. The first decision the students faced in this course was the selection of the "organization", and this selection subsequently influenced 
the corporate "culture" in which the project was to be performed. The past offerings of this project-based course were organized in a manner that was intended to simulate the staged-gate process used in many companies that design and manufacture a wide class of products. A schedule was developed by "management" and key deliverables identified for the engineers. The students were required to organize their time and resources to produce the deliverables consistent with the established schedule. This highly structured approach is quite successful but how does it influence creativity and innovation?

Kelley $^{[5]}$ suggests an alternative process that appears far less structured but is intended to foster innovation. It is based upon the corporate culture practiced at IDEO, a firm specializing in product design and development. This framework for product development appears to be very well suited for the "toys" used in this course. Kelley summarizes the approach that contains five basic steps: 1) Understand the customer, 2) Observe, 3) Visualize, 4) Evaluate and Refine and 5) Implement. Step 4, in particular, relies on the use of prototypes in an iterative manner. The contrast with the more structured approach mentioned earlier and dependence on prototyping in contrast to analytic modeling and simulation presented an interesting alternative that appeared to be well suited for this course.

In preparation for the course the students were encouraged to read Reference 5 as they were notified of this experiment three months prior to the semester. The students were also provided the comprehensive AME470 Inc. employee's manual. This is an approximately 60 page coursepack that describes in detail the deliverables, schedule and other aspects of the project as well as a single page description of the target product, the "an intelligent, interactive toy." Based upon these this information the students were offered the opportunity to accept positions in one of two firms. One job was with an organization AME470 Inc. and the other NDEO. The following highlights the information they were provided describing each opportunity.

\section{$\underline{\text { AME } 470 \text { Inc. }}$}

This represented the organizational structure and operating procedures that are found in many businesses today. Product development takes place in a stage-gate process with well-defined deliverables, milestones and reviews. Key elements of this framework included:

Organization: The product-development team consisted of 4-5 engineers with a project supervisor. The project supervisor (instructor) assigned the team members. The basic project schedule and primary deliverables are indicated in Figure 1 at the end of this paper. The teams had access to "consultants" that could be used to provide support with both technical and nontechnical issues. Each team was required to present a weekly oral briefing on a specified topic to the project supervisor.

Design Methodology: There was an emphasis on engineering analysis, modeling and simulation in the product development process. Corporate technical specialists (staff technicians and teaching assistants) and the project supervisor interacted with the team on a regular basis to assist with answering questions and evaluating progress. Engineering feasibility studies were used to validate or demonstrate various elements of the design and prototypes could be used to validate the engineering studies. 
Salary: (Grade) Individual and group deliverables were specified. Approximately $50 \%$ of the final evaluation was based upon individual performance and the other $50 \%$ upon the performance of the group. The project supervisor provided these evaluations. The group performance was weighted by a confidential peer review. The intent was to evaluate their ability to do "good engineering," effectively communicate and document their efforts and work effectively as part of a team.

Key Deliverables: The team was responsible for a detailed concept product proposal and a concept prototype. Individuals were responsible for an engineering notebook and participation in the formal Concept Design Review. The design proposal was the project's most highly weighted deliverable.

\section{$\underline{\text { NDEO }}$}

This represented a new start-up that would like to make its place in the children's toy product development business. It was very loosely modeled after the IDEO process as described in Reference 5. The key elements of this framework included:

Organization: The engineers were assembled into small self-directed, product-concept development teams. The overall project manager (instructor) was on-site but the studio teams operated in a semi-autonomous fashion. The project teams were self-selected by the members of the engineering staff. Prior to the formation of the studio teams, each employee was given the opportunity to "pitch" a new product concept. Each individual was allowed to select the concept they wanted to pursue.

Design Methodology: A strong emphasis was placed on "prototyping" during concept generation. Analysis, modeling and simulation were to be conducted at the discretion of the team and documented as they deemed most appropriate. Validation of the concept was at the discretion of the design team.

Salary: (Grade) The studio team members were asked to assign their own grades based upon their product and its presentation to the industry panel at the concept design review. The justification for the grades for all members of the team were documented by the team and validated in a written report presented to the project supervisor. The project supervisor then had the prerogative to concur with or take exception to the group's assessment.

Key Deliverables: The product concept for presentation to a panel of industry reviewers was the primary deliverable for this project. The documentation of the project was left to the discretion of the group. Possibilities were suggested that included a "sale's brochure," prototypes, a video, a $\mathrm{CD}$, a formal technical proposal, but it was the sole responsibility of the group to describe and validate their concept and its feasibility to the panel. These deliverables were then used by the team to justify the grades they assigned to the team members.

The students had been given the opportunity to purchase and read Reference 5 during the summer session in preparation for this decision. They were given one day to make their choice of 
firm . Nine students selected the NDEO approach and organized themselves into two design studio teams. Five students selected the AME470 Inc. offer and comprised a single project team. Some of the rationale for these decisions are discussed below.

\section{Integrating Other Discipline Perspectives}

Along with the decision to consider a new class of consumer products as the focus for this project came the realization that the engineering students would need to address issues that had not been considered in previous projects. These included target market assessment and product styling. In recent years some students from the mechanical engineering program had also participated in courses in the College of Business Marketing Department in Product Innovation and some had actually double-majored in Industrial Design in the College of Art and Letters. These students had indicated how those experiences had complemented the ME capstone project and their comments brought about discussions between the faculty involved in each of these programs. The collaboration described herein resulted from those discussions.

The discussions indicated that it might be possible to enhance the learning for students in each of the programs by encouraging collaboration and interaction centered on the engineering product design project. Two undergraduate courses outside of the College of Engineering were identified as opportunities for these collaborations. The Digital 3-D class in the Department of Art, Art History and Design is used to introduce junior-level students to the computer tools for solid body modeling. The Marketing Department has a senior-level course in Product Innovation that, as described in the University Catalog, is used to "introduce students to some important activities and perspectives that can enhance innovation and improve the ability to influence and forecast the adoption and diffusion of innovations."

Both of these courses have their own learning objectives and activities but it was decided that students in each course would be organized into groups and serve as "consultants" to the engineering product design teams. Each of these groups were assigned small projects in their own courses that required them to work with the engineering design teams to gather information and assist in the evaluation of the proposed products. The "design consultants" were tasked with developing 3-D solid models to represent the designs proposed by the engineering teams. Effectively they also served the role of "designers" in suggesting external forms for the products that would enhance their customer appeal. The "marketing consultants" were tasked with performing a limited market analysis on the products proposed by the engineering design teams.

To initiate this collaboration an meeting was held during the first week of class that included students in all three courses. Each of the participating faculty presented ideas related to this collaboration and the students were given the chance to ask questions, play with a selection of "intelligent" toys and eat pizza. Shortly after this meeting, the consulting groups were identified and paired with the engineering design teams. The engineering teams were tasked with providing the marketing and design consultants an initial product concepts at the end of the $3^{\text {rd }}$ week of the project. These initial concepts were to be used by both of the consultant groups as preliminary information to assist them in beginning their respective projects. All additional interactions between the groups were the responsibility of the students and arranged at their discretion. The students were told (in an unrealistic fashion if this was a valid simulation) that their success was 
not dependent upon the input from the other groups. This collaboration had been arranged to assist them in their projects and it was therefore their responsibility to identify and use what information they thought would be the most helpful.

\section{Assessing the Experiment}

The first result of the experiment worth noting involved the selection of the "corporate culture" and the rational for the selection. Prior experience with this course has indicated that group sizes should range from 4-6 students. Nine of the 14 students selected the NDEO offer and five AME470Inc. This was fortunate in that it allowed for one group of five students to be identified with the AME Inc. framework and the remaining nine students were tasked with self-selecting into two studio groups.

The process of selecting groups for the NDEO framework was conducted by the students and it was based upon interests they expressed regarding the type of product that they wanted to address. Five students indicated an interest in working with a "sports" related theme and the other four were interested in some form of a "vehicle." Since the students had the opportunity to consider this project since the end of the previous semester, they had already developed some ideas and used those ideas to help formulate the project groups.

In subsequent discussions with the students, they indicated that the opportunity to set their own schedule and determine what aspects of the project they felt were most important were the main reasons for selecting the NDEO format. Those students who selected the AME470 Inc. culture indicated that the primary motivating factor was that they felt they understood what would be expected from them and they were uncomfortable with the uncertainty associated with the other environment. And, somewhat ironically, some NDEO team members expressed frustration over not knowing exactly what was expected from them.

A number of sources were used to assess the impact and effectiveness of the capstone course presentation as described in this paper. The student's efforts, both in terms of the proposed product and their ability to communicate their efforts in both written and oral forms, were evaluated. The industry panel provided input based upon their participation in the Concept Design Review. The instructors from the two participating programs were asked to comment from their perspective on the collaboration and the students themselves were given a number of opportunities to provide comments and observations.

All three groups developed a product concept, fabricated a prototype that manifested select attributes and behaviors of the proposed product, documented their design in a project report and presented a formal design presentation to a panel of industry reviewers. Thus the fundamental goals of the project were achieved. The concepts that the three teams selected were:

1. AME470 Inc. - A radio controlled surface-vehicle with water cannon.

2. NDEO1 - A trainer and pitching game for little league baseball players.

3. NDEO2 - An interactive radio controlled vehicle for young children.

Each product included an embedded microprocessor and manifested some form of interaction with the user. At the end of this paper Figures 2-7 illustrate some of these product concepts. Additional details can be seen on the web site indicated in Reference 6 . The purpose of the 
current paper isn't to evaluate the concepts but to determine the influence of the structural changes in the course's offering on the student's experience. The following paragraphs address a number of these issues. The obvious difficulty in making this assessment is the lack of a control or comparable benchmark information on previous course formats but the information provided by the students and evaluators has proved useful.

\section{$\underline{\text { Overall Learning Objectives }}$}

On the first day of the course, the learning objectives outlined above were presented and discussed. On the last day the students were asked to complete a multi-page assessment document that asked them to determine their response as to whether these objectives had been met. They were asked to indicate their response on a scale from 0 to 4 - with the 0 indicating that, from their perspective, the goal was not at all achieved and a 4 indicating that the goal was achieved to their satisfaction. The questions and the mean response for the class (in parentheses) are indicted below.

1. It was an experience that required active student participation.(4.0)

2. It enhanced my skills related to engineering design methodology, including modeling, simulation and engineering feasibility studies. (3.0)

3. It simulated the mechanical engineering product development process.(3.0)

4. It illustrated the interaction between competing technical and non-technical issues and the role of compromise, constraints and merit.(3.4)

5. It provided exposure to various phases of the design process, from requirements definition to product realization.(3.5)

6. It helped develop an understanding of the planning, coordination and communication required in a team effort.(3.7)

7. It allowed me to be innovative.(3.6)

Once this written assessment was completed a discussion ensued to review their comments and provide additional information. A number of the more significant points resulting from these discussions are presented herein.

In many areas the student's attitudes spanned the spectrum of opinions. Just about every feature of the course was "liked" by some and "disliked" by others. To demonstrate this point the students were asked if they would prefer being assigned the product to design or have the opportunity to select the product as was the case this year. Three quotes indicate the diversity of opinions, (1) "it would have been less frustrating if we were given a product to design," (2) "I enjoyed the freedom we had in being able to choose our product," and (3) "regardless of whether the product was assigned or not, the students will complain." Trying to discriminate between attitudes expressed "in the heat of battle" and the long-term effectiveness of the educational experience is an ongoing challenge.

\section{Corporate Culture}

Though most preferred the culture they selected, in each case some students wished they had selected the other option. Two of the five AME470 Inc. group would have selected the other 
"job," though just one of the NDEO group members would have switched. Though most of the NDEO team members liked that format, there were specific features that they didn't feel were effective. There was a consensus that, as much as they appreciated the freedom provided to define their own schedule, they wanted to be given clearer details on "just what was expected from them." The one NDEO participant who would have preferred the other option indicated he "needed a more structured schedule." All of the students carried four or five other courses and a number of them indicated that the self-discipline necessary to operate in the NDEO framework created problems in that there was the tendency to let the schedule "slip." Since they were in control of the schedule and deliverables and the only hard deadline was the final presentation, they were inclined to let certain parts of the project, like the documentation, go until the last minute. One noted, "sticking to the schedule was difficult, since we didn't have anyone forcing us to." It was interesting to observe that even though they were given unlimited freedom in deciding how best to document their efforts, both NDEO groups did appear to follow many of the guidelines provided to the AME470 Inc. group. This probably resulted from the fact that they felt these were the ones that would most likely satisfy the "instructor." With the limited practical experience that the students carry into this type of course, it appears that they feel most comfortable with some "templates" to guide their efforts but they do appreciate some level of autonomy. Achieving an effective balance in this area will be the goal in future efforts.

The primary observation associated with the AME470 Inc. team members was that they felt they were expected to do "more work" and were held more accountable for their efforts. During the semester they were required to make weekly progress presentations that they thought were of limited value, at the time, but by the end of the semester they were considered to be quite useful in preparing for the formal Concept Design Review. As one would expect, the perception that one format required more work than the other is quite critical in the academic environment and would probably influence future attempts to "mix" cultures in the same course. The students were asked to maintain weekly records of the time spent on the course and the nature of those activities and these did indicate that the AME470 framework group did spend on the average about $20 \%$ more time per week on this course. In this case, much of this group's effort was dominated by one individual so an accurate assessment of the impact the format had on the student's level of effort is still a matter of concern.

$\underline{\text { Interaction with Marketing and Industrial Design }}$

This feature of the course was met with mixed reactions. In general all the students felt it enhanced each course but that there were issues associated with the implementation. Most of these concerns revolved around relative expectations, scheduling and communication. Where the product development project was the central focus of the engineering capstone design class, it represented only a relatively small "assignment" in the other courses. Though attempts were made to schedule the activities in all three courses in a manner that they would complement each other, some problems were still encountered.

The Industrial Design students were faced with the challenge of applying a new software package and it turned out that the learning curve was steeper than expected. Thus they were able to provide discussion and consultation and some sketches in a timely fashion but detailed computer rendering were not available in a time frame that was of value to the engineers. 
Though the initial goals for this aspect of the collaboration were not met, the fact that the engineers were constantly reminded of the "aesthetic" appeal requirement for their product was of value and it did complement the design experience.

The Marketing students were asked to rate on a scale of 0-4 (4 being the best) the effectiveness of the interactions between them and the engineering and graphic design students. The interaction with engineering was rated as 2.33 and with graphic design as 1.73 . In most cases the issues were related to the difficulty in communicating with the other disciplines and an inadequate set of expectations. One student summarized the initial interaction as " a friend of mine in the class said to me, 'well, we'll make the product and you guys are responsible for the advertising and stuff, right? - he didn't really think we did anything beyond creating ads." Another commented, "they didn't verbalize their needs (in reference to the engineers) or concerns. They would just get frustrated." Putting those two comments in context, this aspect of the course did expose all the students to a different way of thinking. One industrial design student commented, "we learned that effective decision making could only occur with close interaction of all groups." In hindsight one of the greatest benefits of this experiment may have been the fact that it provided an opportunity for those outside of the College of Engineering to get some idea as to how engineers "think" and act.

\section{Selection of the Product Topic}

In general the topic was well received by the students. The one problem that was created by this topic was the perception that the development of a "concept" toy limited their use of their newly evolved "engineering skills." Even though each of the proposed products did contain a number of interesting and technically demanding mechanical elements most could be ignored in the concept development phase. Subsequent discussions indicated that it was not so much the fact that their product didn't require them to apply their skill-set but in most cases they were unsure how to do so in an efficient manner. Most of the engineering students felt that they could relate to the product and developed a strong interest in their eventual concept. In a number of cases an attitude that one might expect was manifest, "It was a good topic but... I would have liked something without so many safety and marketing issues to worry about." Addressing such an attitude was actually something one might consider a valid reason to deal with a similar class of consumer products in the future. In general they were able to relate to the product and it's performance, through their own experiences and those of younger siblings.

Regarding the issue of innovation and creativity, this product class did appear to provide the opportunity to explore and develop new ideas. The industry review panel was particularly impressed with the actual results of the student's efforts. In one case Fisher-Price brought to the 2001 Christmas market a toy with some of the basic attributes of the student's design, the NDEO2 group, and this new toy was announced well after the students had begun their efforts and defined their concept.

Evaluating the Student's Performance - i.e. Grades!

One of the least positive aspects of the experience was the attempt to get the students who participated in the NDEO framework to decide what would be the most effective manner to 
document and validate their product concepts. Since the teams had unlimited freedom in selecting their concepts, it was difficult for the instructor to define $a$ priori how best to document their ideas. They were provided examples of previous product proposals for student projects but attempts to acquire "real world" documentation for similar products proved unsuccessful as this information is typically proprietary. The lack of good templates to help guide their efforts was problematic.

Since the AME470Inc framework had been used for many years the student assessment mechanisms have been tested and proven to be effective. Some percentage of the student's grade is based upon individual performance and the remaining upon team performance. There are nine different graded events during the project and thus the ability to differentiate and identify an individual's contribution is present.

The manner in which the grades were proposed to be assigned to the NDEO group is described above. The students were given the opportunity to select their own deliverables and at the end of the project they were tasked with preparing a written self-assessment. In most cases the instructor agreed with the student's self-assessment but in those cases where they disagreed, the instructor did not have the documentation necessary to differentiate the individual's performance from that of the group. This presented a problem in two cases where the group had performed very well but the individual's contribution was suspect. This particular experiment was deemed less than satisfactory and would not be repeated.

\section{$\underline{\text { Participating Faculty Responses }}$}

The paper documents the impressions of the mechanical engineering design instructor but other comments regarding the experience from the other faculty involved in this collaboration will help shape future efforts. The instructor from the industrial design program noted that the identification of groups should happen sooner in the semester with a collaborative meeting with individual groups and assigned partner groups getting together in a social atmosphere. Due to enormous activity load of the students, he discovered that physical meetings would not occur unless assigned. He noted that when the art students were asked about the team interactions he received responses like "Engineering says this...." and "Marketing says this..." instead of identifying individuals and personnel involved in these areas. He felt that communication was not particularly effective and without formally assigned meeting times the project was hampered by a " they're not ready, "or "they have not called us back," or "I sent an e-mail "syndrome. He felt that it would have been preferred if there had been an initial "workshop" to form groups, and have initial brain-storming exercises for the interdisciplinary team members. He suggested this involve a verbal and visual activity session that would encourage all to identify, draw and articulate a product image, and to examine function, market, and manufacturability, early on in the process. As indicated above, the art students that formed this collaboration were actually participating in a course designed to instruct them in the use of a particular computer application. It has been suggested that an industrial design studio project course would provide a much better group for this collaboration.

The marketing instructor indicated that this had been a positive experience for his students but he too felt that better coordination and scheduling of deliverables between the student groups was 
necessary. This first attempt to build this collaboration was hampered by "already scheduled" courses and a desire to experiment without major changes to existing class structures. Future efforts will require better coordination and the requirement that on a somewhat regular basis the student collaborators meet. A more important observation was related to the role of marketing and its involvement earlier in the process. The marketing students should be engaged in articulation of the customer's needs and work more closely with the engineers and industrial designers during the first phases of concept selection.

\section{$\underline{\text { Industry Responses }}$}

The industry review panel at the Concept Design Review serves two purposes. First is to provide direct feedback to the student groups regarding their product and their ability to present the results of their efforts. The reviewers provide written comments that are then shared and discussed with the students. They are asked to comment on the overall organization, the product description, the student's ability to demonstrate the technical feasibility of the product, the effectiveness of their visual aids and their ability to answer questions.

The following is one of these responses:

"Overall organization:

Right from the start, this team had punch. From the effectiveness of the brochure, to the 'design of the experience', these guys were focused, direct and on a mission.

Product description:

Clearly they could identify with the user and were very sensitive to the user's perspective and the value in the performance, visual styling and interaction. Another point that was not lost was understanding and using the learned behavior and appeal of the Nitendo controllers and designing the controller to match this intuitive (for that age group!) interaction.

Technical feasibility:

Using off the shelf technology, they took the route of implementing an idea rather than implementing the solution. This is critical in understanding the value of that idea and not being married to the hardware or technological features. Good sensor analysis and it showed the value of building something and learning, and building again.

Visual aids:

In the presentation they pointed out their concerns and then addressed them one by one. Here's our concern... and this is what we think...our next concern is..... Also, they demonstrated a good balance between process, strategy, and dipping into the details occasionally.

Questions:

Good and direct, and by the overall reaction of the panel, it was easy to engage in conversation with the team, showing that we (the panel) clearly connected with the presentation."

There were four reviewers at the CDR and all their comments were provided to and discussed with the student team. In most cases they were positive and reinforced the student's efforts. They did highlight certain other issues, for example, "some testing data would be helpful, for instance, the raw output of a sensor and the difficulties in conditioning the signal, the repeatability and 
accuracy ..." These helped reinforce some issues brought to the student's attention by instructors but when they come from "outside sources", they immediately possess more credibility.

The reviewers also provide comments on the overall experience. This year they appreciated the attempt to interface the three diverse disciplines and understood the difficulties of doing so in the academic environment. Both industry reviewers commented on the innovative nature of the products proposed by the students. They also emphasized the importance of the role of prototyping and marketing for this particular class of products and helped all involved develop a better understanding of the complexity of the interactions that occur between these disciplines.

\section{Final Comments and Future Plans}

Developing an effective capstone design experience is an ongoing challenge. Providing engineering students with an experience that is both consistent with their emerging skill set, resources and schedules, but also one that effectively prepares them for their careers is difficult. Many of the elements of this experiment, as noted in the paper did appear to enhance the learning experience, whereas some did not. Continued efforts will be made to integrate various discipline perspectives into the capstone design experience. It is felt that not only will this improve the learning for the engineering students but also provide the opportunity to introduce the engineer's viewpoint to students outside the discipline. Some compromise between the two corporate cultures considered herein seems to be best suited for the students at Notre Dame, and this may be institutionally dependent. Providing freedom that allows for innovation is important but this must be done in a manner that provides the students with reasonable guidelines and milestones. One of the most exciting and rewarding aspects of teaching "design" is that, just as there are no right-answers for the product, there are no right-answers for the process and instructors are able to experiment and learn what best suits their teaching style and the institutional goals.

\section{Acknowledgments}

The activities detailed in this paper were made possible through the contributions of many individuals. First and foremost the students who participated in the course during the Fall 2001 semester, the participation and support provided by Fisher-Price (Tony DeSimone), and IDEO (Jim Tappel and Tracy Lukawski), the contributions of Dr. Joseph Guiltinan, Professor and Chair, Marketing Department in the Mendoza College of Business, Dr. John Caruso, Professor of Art, Art History and Design, the advice and support provided to the student design teams by Mr. Greg Brownell, microprocessor technician in the Department of Aerospace and Mechanical Engineering, and Dhanesh Padmanebhan, Neil Petroff and Weiyu Liu, Graduate Teaching Assistants in the Department of Aerospace and Mechanical Engineering.

\section{References}

1. Schmaltz, K., Duesing, P., "Lessons Learned from Teaching Industry-Based Senior Projects," Proceedings of the 2001 ASEE Annual Conference and Exposition, Albequeque, New Mexico, June 2001.

2. Abu-Mulaweh, H., Younis, N., "Local Industry Involvement in the Support of Capstone Design Projects," Proceedings of the 2001 ASEE Annual Conference and Exposition, Albequeque, New Mexico, June 2001. 3. Simpson, T., et al. , "IME, Inc. - A New Course for Integrating Design, Manufacturing and Production in the Engineering Curriculum," Proceedings of the 2001 ASEE Annual Conference and Exposition, Albequeque, New Mexico, June 2001. 
4. Hanson, A., et al. , "Integrating the Real World into the Capstone Experience," Proceedings of the 2001 ASEE Annual Conference and Exposition, Albequeque, New Mexico, June 2001.

5. Kelley, T., Littman, J., The Art of Innovation, Currency Books, New York, New York, 2001.

6. WWW site for AME470, Senior Design Project, http://www.nd.edu/ batill/www.me470/, Last accessed Jan. 2002.

\section{STEPHEN BATILL}

Professor of Aerospace and Mechanical Engineering and the Associate Dean for Educational Programs in the College of Engineering at the University of Notre Dame. His teaching and research interests are in the area of multidisciplinary systems design and optimization.

\begin{tabular}{|c|c|c|c|c|c|c|}
\hline \multirow[b]{2}{*}{ ID } & \multirow[b]{2}{*}{ Task Name } & \multicolumn{2}{|c|}{ Qtr 3, 2001} & \multicolumn{3}{|c|}{ Qtr 4, 2001} \\
\hline & & Aug & Sep & Oct & Nov & Dec \\
\hline 1 & Receive RFP & & $/ 01$ & & & \\
\hline 2 & Individual Concept Develpmnt & & & & & \\
\hline 3 & Individual Concept Memo & & & & & \\
\hline 4 & Team Concept Refinement & & & & & \\
\hline 5 & Team Concept Selected & & & & & \\
\hline 6 & Team Project Planning & & & & & \\
\hline 7 & Project Plan Review & & & & & \\
\hline 8 & Group Concept Design Study & & & & & \\
\hline 9 & Feasibility Study Prop. Dev. & & & & & \\
\hline 10 & Feasibility Study Prop. Completed & & & & & \\
\hline 11 & Indiv. Eng. Feasibility Study & & & & & \\
\hline 12 & Eng. Feasibility Study Completed & & & & & \\
\hline 13 & Draft Proposal Preparation & & & & & \\
\hline 14 & Draft Proposal Completed & & & & & \\
\hline 15 & Proposal Re-write & & & & & \\
\hline 16 & Design Review Preparation & & & & 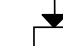 & \\
\hline 17 & Prototype Completed & & & & & 29/01 \\
\hline 18 & Design Review Rehearsal & & & & & $/ 29 / 01$ \\
\hline 19 & Concept Design Review & & & & & $12 / 6 / 01$ \\
\hline 20 & Final Proposal Due & & & & & $12 / 6 / 01$ \\
\hline
\end{tabular}

Figure 1. Project Schedule and Major Milestones 


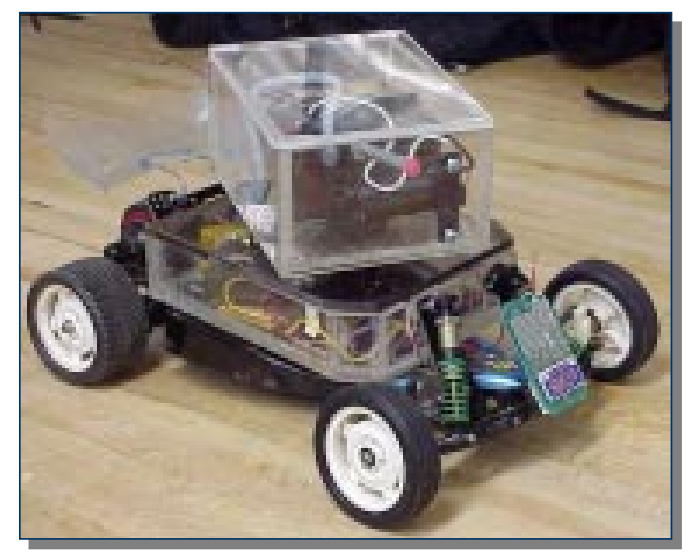

Figure 2. Prototype Water Cannon Vehicle

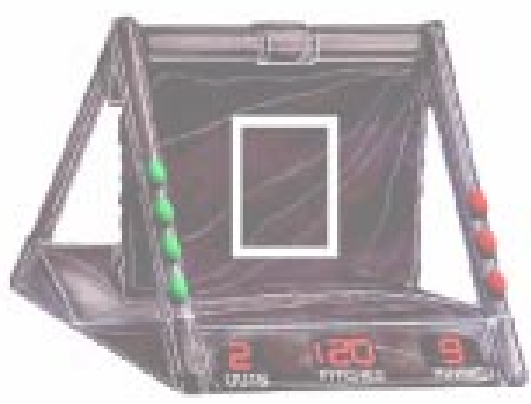

Figure 4. Designers Concept for the Pitcher Training System

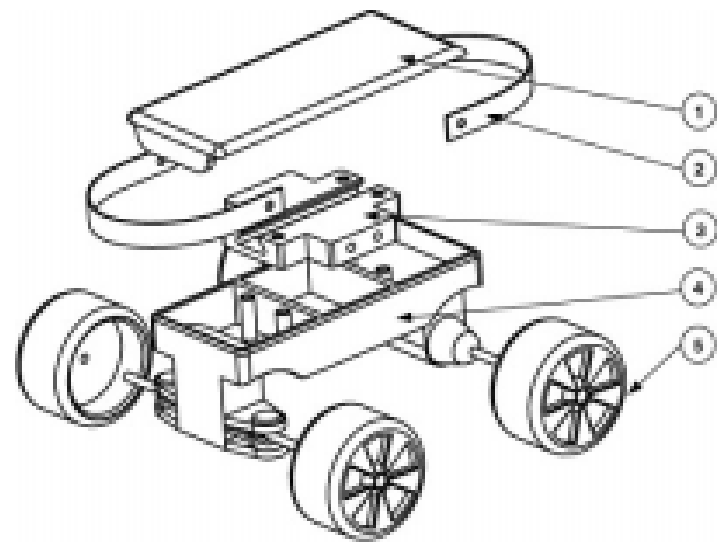

Figure 6. Assembly for Basic Chassis of the Interactive Vehicle

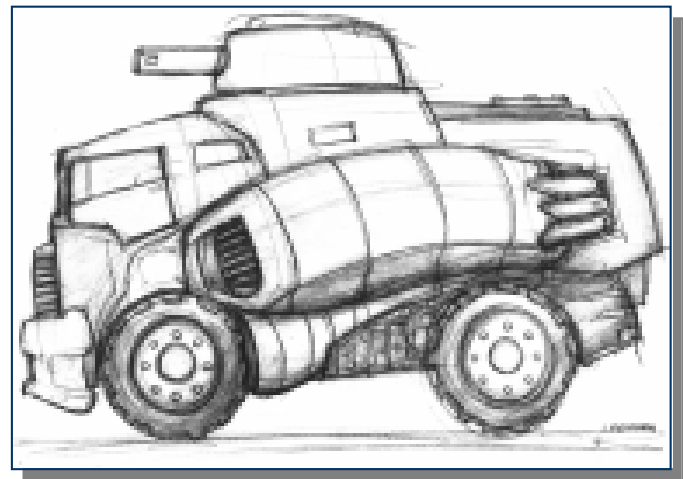

Figure 3. Designers Concept for Water Cannon

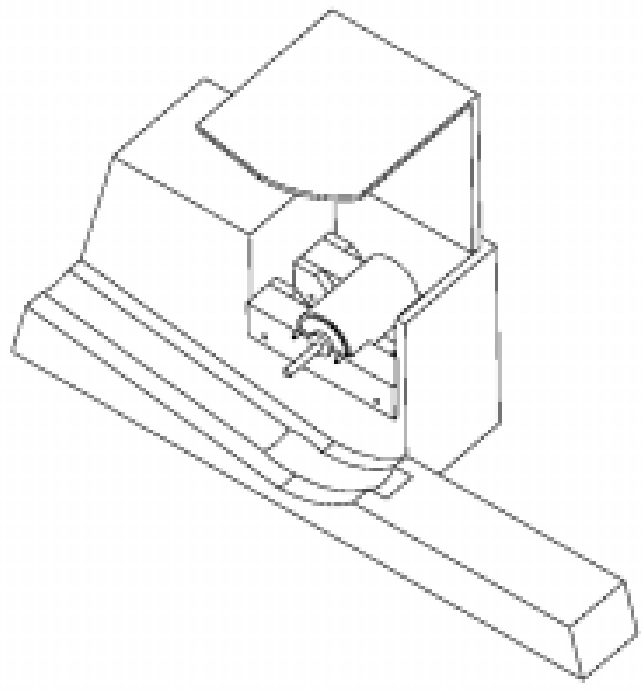

Figure 5. Ball Throw-back Mechanism for the Pitcher Training System

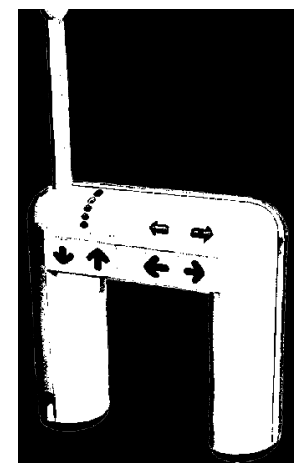

Figure 7. Controller for Interactive Vehicle Concept 